\title{
Current Approaches to the Treatment of Post-Stroke Aphasia
}

\author{
Julius Fridriksson, ${ }^{\mathrm{a}}$ Argye Elizabeth Hillis ${ }^{\mathrm{b}}$ \\ aDepartment of Communication Sciences and Disorders, University of South Carolina, Columbia, SC, USA \\ ${ }^{b}$ Department of Neurology, Johns Hopkins University School of Medicine, Baltimore, MD, USA
}

\begin{abstract}
Aphasia, impairment of language after stroke or other neurological insult, is a common and often devastating condition that affects nearly every social activity and interaction. Behavioral speech and language therapy is the mainstay of treatment, although other interventions have been introduced to augment the effects of the behavioral therapy. In this narrative review, we discuss advances in aphasia therapy in the last 5 years and focus primarily on properly powered, randomized, controlled trials of both behavioral therapies and interventions to augment therapy for post-stroke aphasia. These trials include evaluation of behavioral therapies and computer-delivered language therapies. We also discuss outcome prediction trials as well as interventional trials that have employed noninvasive brain stimulation, or medications to augment language therapy. Supported by evidence from Phase III trials and large meta-analyses, it is now generally accepted that aphasia therapy can improve language processing for many patients. Not all patients respond similarly to aphasia therapy with the most severe patients being the least likely responders. Nevertheless, it is imperative that all patients, regardless of severity, receive aphasia management focused on direct therapy of language deficits, counseling, or both. Emerging evidence from Phase II trials suggests transcranial brain stimulation is a promising method to boost aphasia therapy outcomes.
\end{abstract}

Keywords Language therapy; Aphasia; Speech therapy; Stroke; Brain

\author{
Correspondence: Argye Elizabeth \\ Hillis \\ Department of Neurology, Johns \\ Hopkins University School of \\ Medicine, Phipps 446, 600 N. Wolfe \\ Street, Baltimore, MD 21287, USA \\ Tel: +1-410-955-2228 \\ Fax: +1-410-614-9807 \\ E-mail: argye@jhmi.edu \\ https://orcid.org/0000-0002-5192- \\ 1171
}

Received: December 16, 2020

Revised: February 21, 2021

Accepted: April 30, 2021

\section{Introduction}

Aphasia is a language disorder that can have profound negative effects on quality of life. It is most often due to left hemisphere stroke but can also be caused by other types of damage-such as trauma or neurodegenerative disease-that affect the language network of the brain. Here, we review therapy for post-stroke aphasia, focusing on recent clinical trials. Although most neurologists have the greatest familiarity with pharmaceutical interventions, we begin by discussing behavioral therapies, because other interventions augment behavioral inter- ventions. That is, medications and noninvasive brain stimulation have been used in conjunction with, rather than in place of, behavioral therapies.

Several recent publications have reviewed the mechanisms of aphasia recovery, and in some cases the mechanisms of therapy ${ }^{1-4}$ revealed by changes in task-related brain activations or changes in functional connectivity within functional networks. ${ }^{1-6}$ Therefore, we will emphasize the empirical effects of therapy with less focus on the neural mechanisms that promote treated recovery from aphasia. 


\section{Behavioral interventions}

\section{Behavioral therapy to improve language in stroke aphasia: the basics}

By far, the most common approach to aphasia rehabilitation is behavioral speech and language therapy (SLT). SLT was even described by Paul Broca in his seminal 1865 paper, $^{7}$ and it remains the standard of care for patients with aphasia. ${ }^{8}$

There are many different approaches to SLT, with two main camps: (1) Impairment-based approaches focus directly on decreasing the language impairment through structured therapy that targets the sub-components of language such as phonology, lexical-semantics, or syntax..$^{9-11}$ The goal is to improve language functions with the assumptions that doing so will generalize to communication abilities and, by extension, communicative quality of life. (2) Functional communication approaches more directly target communication abilities and do not necessarily focus on generalization to reduce speech or language deficits. Rather, these latter approaches are more likely to focus on stimuli with direct personal relevance. Additionally, functional therapy emphasizes eliminating communication barriers in the environment, caregiver training to enhance communication, and improving the success of communication rather than reducing impairment (see Martin et al. ${ }^{12}$ for review and examples of the two approaches).

\section{Efficacy of behavioral speech and language therapy for aphasia}

The literature on aphasia therapy is marred by mostly single-case design and small group studies (e.g., references ${ }^{13-15}$ ). Perhaps because of this historical focus on underpowered studies, the efficacy of aphasia therapy was debated for decades and, until relatively recently, remained highly controversial. ${ }^{16-18}$ Furthermore, by focusing solely on speech and language measures as markers of outcome, most studies failed to demonstrate meaningful improvements. In acute patients, it has been relatively difficult to demonstrate that early aphasia recovery is spurred by SLT rather than inherent recovery processes. ${ }^{19}$ In addition, no standards have been developed for what works best for patients with different aphasic impairment patterns. However, in the past decade, a much clearer picture of the overall efficacy of SLT for aphasia has emerged. Meta-analyses of a large number of smaller studies generally support the notion that SLT is very much worthwhile for aphasic patients. ${ }^{16,17,20,21}$ Moreover, the results of a recent Phase III randomized controlled trial (RCT) by Breitenstein et al. ${ }^{18}$ unequivocally support the use of impairment-based SLT to improve speech production in chronic aphasia. Specifically, this trial revealed that SLT does improve effectiveness of verbal communication measured using the Amsterdam-Nijmegen Everyday Language Test $A$ (ANELT-A ${ }_{i}^{22} A$-scale [Cohen's $d=0.58$; medium effect size]). Moreover, the trial also revealed an improvement in communicative quality of life (Cohen's $d=0.27$ ), an important finding that had eluded many earlier trials (e.g., references ${ }^{23-25}$ ). Taken together, large meta-analyses and the Breitenstein et al. ${ }^{18}$ trial provide robust evidence in favor of SLT for post-stroke aphasia.

\section{Optimal timing of intervention: chronic vs. acute phase of post-stroke}

In spite of progress in the field, the optimal timing of intervention after stroke remains unclear. Equally important, it is not known if or when patients reach a recovery plateau. Whereas animal models of stroke suggest early intervention is probably important ${ }^{26}$ we have no definitive data in aphasia. ${ }^{27}$ Although most aphasia therapy studies have enrolled chronic patients, it seems likely that earlier aphasia therapy is also effective. ${ }^{28}$ However, two large RCTs published in the last 5 years do not support this notion. In the Rotterdam Aphasia Therapy Study-3 (RATS-3), ${ }^{29} 152$ patients with acute aphasia due to stroke were randomized to receive either 4 weeks of impairment-based SLT (1 hour/day) or no therapy. Relying on the same outcome measure as Breitenstein et al., ${ }^{18}$ ANELT-A ${ }_{1}^{29}$ no group differences were revealed at the primary endpoint $(P=0.805)$, measured at 4 weeks after randomization, or at secondary endpoints at 3 $(P=0.767)$ and $6(P=0.807)$ months after randomization. A more recent RCT by Godecke et al. ${ }^{30}$ titled Very Early Rehabilitation for SpEech (VERSE) also yielded a non-significant result for the primary analysis. Among 246 patients with acute aphasia, there were no differences in changes in overall aphasia severity between the three study arms $(P=0.59)$ : usual care, usual care +20 SLT sessions, and usual care +20 sessions of specialized impairment-based therapy program designed by an "aphasia Expert Advisory Committee." The mean number of therapy hours in the 'usual care' group was 9.5 (standard deviation [SD] 7.6) over 28 days whereas the other two groups averaged 22.7 hours of therapy (SD 8.4) over 32 days. Although VERSE did not include a 'no therapy' control arm, it suggests that increasing the intensity of SLT from a mean of 0.34 to 0.71 hours/day in the early phases of recovery may not further improve recovery from aphasia. If there is a dose-response relationship between the amount of therapy and early recovery from aphasia, one would expect that the more intensive groups would have experienced better outcome. In this context, it is worth noting that early aphasia recovery is highly variable and is probably influenced by several different factors that may be difficult to control in rehabilitation studies. Equally important, it is clear that not all SLT 
approaches are equally potent and that future trials employing different kinds of SLT may reveal positive outcomes. Moreover, future research may identify the minimum dose needed to affect language improvement in acute to subacute stroke. Just like there is considerable variability in response to $\mathrm{SLT}_{1}{ }^{31}$ it seems reasonable to suggest that some patients may need more therapy to experience an improvement in communication abilities.

In contrast to early aphasia therapy, there is ample evidence, including Breitenstein et al., ${ }^{18}$ that more SLT in the chronic phase is associated with greater long-term recovery. A recent study revealed that among chronic stroke patients, improvement in language processing over several years in language processing among chronic stroke patients with aphasia is associated with more SLT sessions. ${ }^{32}$ This study emphasized that approximately half of chronic stroke patients continue to experience recovery from aphasia even many years after stroke. The remaining half either are relatively stable or actually experience decline, an often overlooked fact that may represent an under-appreciated therapeutic target. Two earlier studies very much echo the finding that approximately half of chronic patients continue to experience recovery. ${ }^{33,34}$ Reasons for decline in language by other patients remain unclear, but white matter hyperintensities seen on T2-magnetic resonance imaging (MRI) or fluid attenuated inversion recovery may be one culprit. One study revealed that patients who experienced long-term language decline were more likely to show greater extent of white matter hyperintensities in comparison to patients who stabilized or improved. ${ }^{35} \mathrm{~A}$ related study showed that these white matter hyperintensities are disproportionally associated with loss of long-range white matter fibers, which could provide a more specific mechanistic account of worsening aphasic symptoms in chronic patients. ${ }^{36}$ Although some studies indicate that on average, stroke patients show steady cognitive decline after stroke, ${ }^{37}$ a careful review of the literature indicates that the average slow decline instead reflects a sharp decline by about $25 \%$ of patients, but slow improvement or stability in others. The finding that approximately a quarter of individuals with post-stroke aphasia are at risk for significant decline in function emphasizes the need for more aggressive monitoring of, and therapy for, vascular risk factors among these patients.

\section{Predicting the outcome of behavioral therapy of aphasia}

Although demonstration for the efficacy of SLT for aphasia is improving, predicting which patients are likely to respond and what therapy works best for individual patients remains a major challenge. In 1972, Darley ${ }^{38}$ suggested that factors such as age, education, intelligence, social status, health, time post-stroke, and aphasia type are related to success in aphasia therapy, but that very few studies had actually incorporated these factors as predictors of outcome. Unfortunately, more than four decades later, the situation is not drastically improved, as research has failed to establish a strong relationship between specific patient factors and treated aphasia recovery. A few studies have related patient characteristics to spontaneous recovery $i^{14,39-41}$ however, it is not known whether the same factors would predict aphasia therapy outcome. Accordingly, clinicians who routinely treat patients with aphasia have somewhat limited empirical data to guide their therapy and predict outcome. Whereas biomarker studies are common in medicine, somewhat limited research has focused on understanding the relationship between patient factors and aphasia rehabilitation potential in stroke (e.g., references ${ }^{40,42,43}$ ). Some recent studies suggest imaging markers, such as larger lesion volume $e^{44,45}$ and greater severity of leukoaraio$\mathrm{sis}_{1}{ }^{35}$ are associated with worse long-term outcome. Other factors have also been implicated such as the degree of damage to posterior superior temporal gyrus or arcuate fasciculus, ${ }_{1}^{46,47}$ independently of lesion volume, and recovery in patients with damage to these critical areas may be influenced by medications. ${ }^{46}$

Aphasia severity, a factor related to overall lesion volume, is one of the very few variables that has been identified as a reliable predictor of SLT outcome, and it is generally accepted that more severe patients are less likely to respond to SLT. ${ }^{41,48}$ The aforementioned trials by Breitenstein et al. ${ }_{1}^{18}$ Nouwens et al., ${ }^{29}$ and Godecke et al. ${ }^{30}$ each revealed that aphasia severity was a strong predictor of overall outcome with more severe patients benefitting less from SLT. It is a caveat; however, that aphasia severity is a multidimensional construct where different patients with severe aphasia might present with very different language impairment profiles. Nevertheless, all else being equal, patients with more severe language impairment are probably less likely to experience spontaneous or therapy-induced recovery. Importantly, speech-language pathologists seem to be very much aware of the connection between aphasia severity and treatment outcome. In a survey of 54 speech-language pathologists, severity and nature of post-stroke aphasia were identified among the most important factors for forming an aphasia prognosis. ${ }^{49}$ We will return to this aphasia severity and treatment outcome in more detail below.

In a trial that recently completed enrollment $(n=128)$ titled Predicting Outcomes of LAnguage Rehabilitation in aphasia $(P O L A R)^{50}$ some initial results are emerging, identifying factors that influence SLT-related improvements in naming and discourse production at 1-week, 1-month, and 6-months following SLT completion. POLAR involves a cross-over design where half of the participants with chronic aphasia are randomized to first 

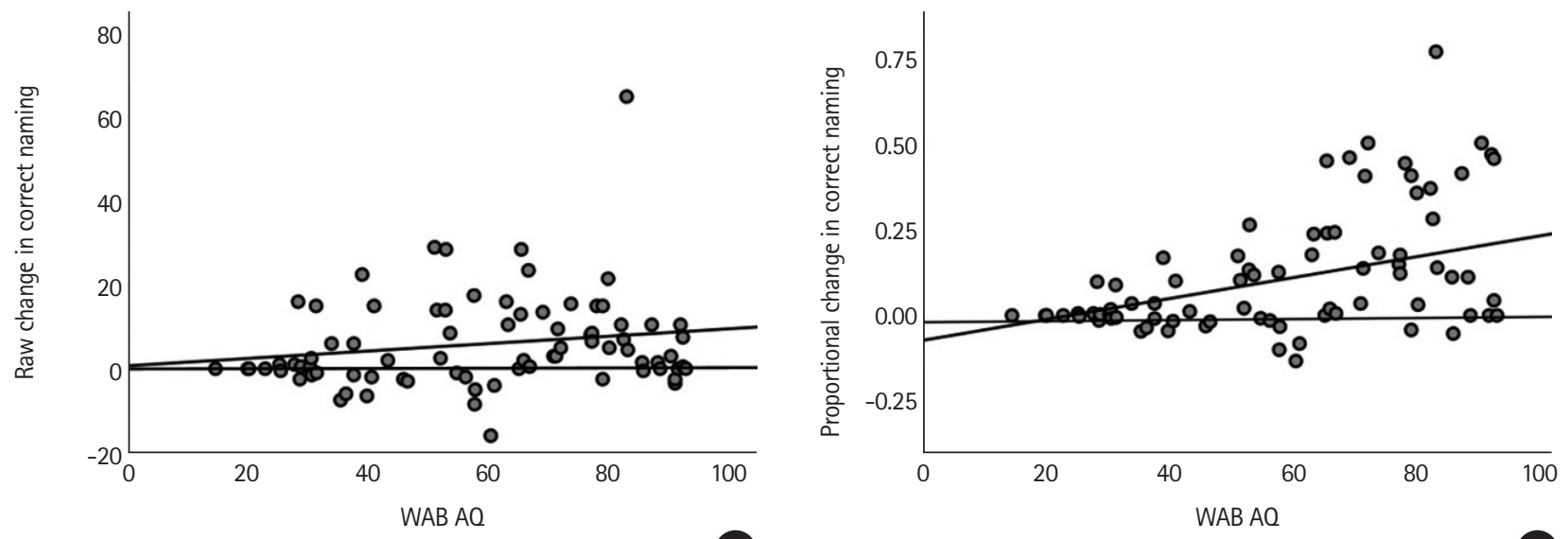

A

Figure 1. The relationship between improvement in naming (y-axis) and aphasia severity (x-axis). Improvement in correct naming was measured using the Philadelphia Naming Test (PNT) whereas aphasia severity was qualified as the overall severity score, aphasia quotient on the Western Aphasia Battery-Revised Aphasia Quotient (WAB-R AQ). (A) The graph shows raw change in correct naming whereas (B) the graph shows change in naming as proportion of potential room for improvement. Note the discrepancy in the graphs at the higher end of the WAB AO spectrum, which is caused by the mildest cases having relatively limited room for improvement at baseline compared to their counterparts with more severe forms of aphasia.

undergo 3 weeks of SLT (5 times/week) focused on phonological processing and then receive 3 weeks of semantically focused SLT with a 4-week break between the two SLT phases. The remaining half of participants are randomized to receive semantic SLT first and then phonological SLT during the second therapy phase. Preliminary analyses incorporating data from the first 74 trial completers revealed statistically significant improvements in correct naming on the Philadelphia Naming Test (PNT) ${ }^{51}$ when comparing results between baseline and 1-week after completion of the second therapy phase $(t(74)=6.1765, P<0.00001)$. This effect was maintained at 6 months after therapy completion $(t 74)=5.6, P<0.0001)$. In addition, significant improvements were revealed in discourse production immediately after completion of the second therapy phase $(t(74)=2.37, P=0.02)$, and again at 6 months post-therapy $(t(74)=3.23, P=0.002)$. Discourse production was qualified and measured as total words produced per minute during three separate discourse tasks.

In accordance with the primary purpose of POLAR, linear mixed effects modeling was applied to understand what baseline factors predict therapy outcome at 6 months post-treatment. For change in correct naming, two predictors emerged: Overall aphasia severity $(P<0.03)$ and participant age $(P=0.006)$. Overall, older individuals and those with more severe aphasia were less likely to show treatment related improvements in the POLAR trial. A significant relationship between a measure on a cognitive test that is thought to minimally tax with involve language processing, the WAIS matrix test and treatment outcome in POLAR was not revealed, which is inconsistent with what others have found. For example, Dignam et al. ${ }^{52}$ revealed that cognitive factors such as verbal working memory predict
SLT-related improvements in naming. Similarly, a study by Gilmore et al..$^{53}$ suggested that cognitive factors predict the outcome of SLT targeting either sentence comprehension or naming in aphasia. Much like Breitenstein et al.., ${ }^{18}$ Nouwens et al. $^{29}{ }^{29}$ and Godecke et al. ${ }_{1}{ }^{30}$ these preliminary data from POLAR suggest participants with more severe aphasia are less likely to respond to SLT. This point is illustrated in Figure 1, which includes data from the POLAR trial showing the relation between WAB-R aphasia quotient (WAB-R AO), and improvements in correct naming on the PNT from baseline to 1-week post-therapy. The WAB-R AQ is a measure of overall severity, and the therapy outcome here was calculated two different ways (1) raw change in correct naming and (2) change in correct naming divided by the room for improvement at baseline. These data suggest that although milder aphasia does not guarantee positive therapy-related improvement in naming, the most severe patients are considerably less likely to respond. As a side note, overall lesion size was not associated with outcome at 6 months post-therapy in POLAR.

Another POLAR report showed that the type of SLT matters for different individuals with aphasia. Again, POLAR involves a cross-over design aimed at comparing the effects of phonologically focused SLT to semantically focused SLT on speech production in aphasia. ${ }^{54}$ Regardless of therapy order, the average improvement in naming following the semantically focused SLT was far superior to the outcome of the phonologically focused SLT ( $P=0.008)$. In spite of the clear overall advantage of the semantically focused SLT, it is worth noting that different patterns of therapy response emerged across the two therapy types. Some participants responded to both approaches, some 


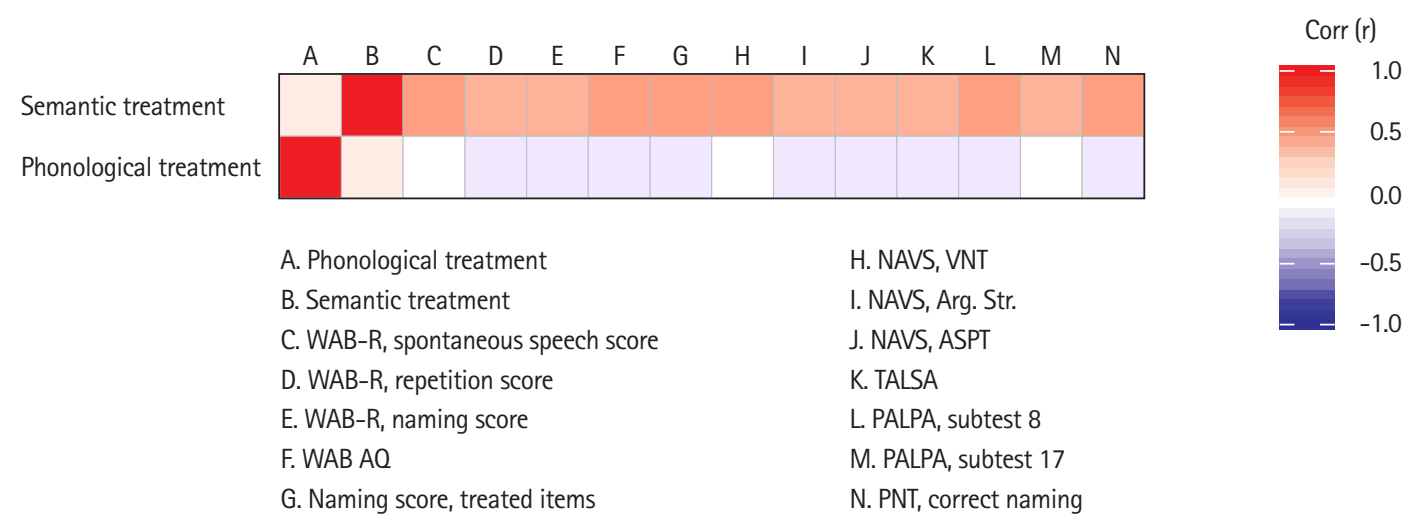

Figure 2. Correlation matrix showing improvements in correct naming following semantic therapy (top row) and phonological therapy (bottom row) in relation to several different language tests. AQ, aphasia quotient; NAVS, Northwestern Assessment of Verbs and Sentences; VNT, verb naming test; Arg. Str., argument structure; ASPT, argument structure production test; TALSA, Temple Assessment of Language and Short-term Memory in Aphasia; PALPA, Psycholinguistic Assessment of Language Processing in Aphasia (Subtest 8: Nonword repetition; Subtest 17: Phonological segmentation of final sounds in words); PNT, Philadelphia Naming Test.

responded to only one and several more showed no response, regardless of SLT type. Crucially, there was an interaction between aphasia severity and therapy type response, where more severe individuals with less fluent speech responded more to phonological therapy $(P=0.049)$, whereas milder cases showed significantly better response to semantic therapy $(P=0.018)$. This relationship is clearly demonstrated in Figure 2 , which shows the correlation matrix between naming improvements following the semantic or phonological therapies in relation to various measures of language abilities. Specifically, positive response (improvement in naming) to the phonologically focused SLT was negatively correlated with various language scores, including measures of grammatical and phonological processing as well as measures of overall aphasia severity and naming impairment. In contrast, response to the semantically focused SLT was positively correlated with the same measures of language abilities. With regard to aphasia type, individuals with either anomic or conduction aphasia showed preferentially better response to the semantic than the phonologically focused treatment. However, no aphasia type was associated with significantly better response to the phonologically focused treatment over the semantic treatment.

To explore whether lesion location predicted outcome in POLAR, lesion-load in language related cortical regions ${ }^{55}$ was used to predict improvement on the PNT $(n=77)$ in univariate analyses. For overall change in correct naming on the PNT from baseline to 1-week after completion of the second treatment phase, greater proportional damage to the left middle occipital gyrus ( $B=-0.294, P=0.010$ ) and the posterior middle temporal gyrus ( $B=-0.216, P=0.060$ ) predicted poorer response to treatment (Figure 3). Response to phonological treatment was

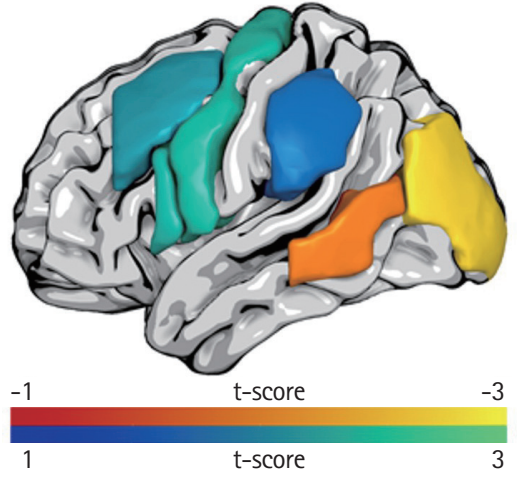

Figure 3. Overall improvement in correct naming on the Philadelphia Naming Test (PNT) was predicted by less damage to the middle occipital gyrus and posterior middle temporal gyrus (red-yellow scale) whereas greater PNT improvement following the phonological treatment phase was predicted by greater damage to anterior regions and the supramarginal gyrus (blue-green scale). The color scales indicate t-scores.

independently predicted by damage to four regions of interest: the middle frontal gyrus $(B=0.232, P=0.042)$, inferior frontal gyrus (IFG) pars opercularis ( $B=0.255, P=0.025)$, precentral gyrus ( $B=0.260, P=0.022)$, and supramarginal gyrus $(B=0.199$, $P=0.083)$. Interestingly, aphasia severity did not emerge as a significant predictor when damage to these four regions was accounted for. No single lesion location predicted response to semantically focused treatment. This preliminary report from POLAR suggests that lesion location is actually associated not only with overall SLT response but also with SLT type. It is too early to say whether these results can be used to guide clinical treatment of aphasia but, at the very least, they provide insights into what kinds of lesion patterns are associated with poor treatment outcome. 
There are certainly many other ways that SLT can be varied other than contrasting semantic versus phonological training. For example, Conroy et al. ${ }^{56}$ compared SLT that emphasized quicker naming to a more conventional approach where speeded naming was not emphasized. In short, this study revealed that greater focus on faster speed and greater accuracy during training was more effective for improving naming accuracy and speed of naming for trained items as well as for generalization to connected speech. Along with Basilakos et al. ${ }^{35}$ the findings by Kristinsson et al. ${ }^{54}$ emphasize the importance of personalized predictors of therapy outcome where baseline factors can be used to stratify SLT type.

In a retrospective analysis, our group (unpublished) examined data from three separate trials to understand the relationship between overall aphasia severity, age, and aphasia therapy success. Although the three studies involved different kinds of aphasia therapy and different sample sizes (total $n=179$ ), the outcome measures for each trial were similar and focused on changes in correct naming abilities. Across all three studies, overall aphasia severity, measured as the WAB-R AQ, was a strong predictor of therapy outcome. Specifically, individuals with more severe aphasia were less likely to respond to SLT. In two out of the three studies, age was also related to outcome, such that older participants were less likely to respond to therapy. Given that greater aphasia severity is, in general, related to larger lesion size, it seems possible that lack of improvement among more severe patients is related to less residual cortex that can take over the function that was lost. Consistent with previous research by Bonilha et al. ${ }^{57}$ greater sparing of the residual language network is related to better SLT response. The relation between younger age and better SLT response is certainly not surprising given that any kind of speech and language restoration associated with SLT must be related to either functional or structural plasticity, or both. As has been shown in numerous studies, the extent of brain plasticity decreases steadily with age ${ }_{1}^{58-60}$ which may explain why older patients are somewhat less likely to respond to SLT. However, it is imperative to note that although age was related to outcome, it was a relatively weak predictor compared to overall aphasia severity. Indeed, many participants older than 65 in the aforementioned studies did respond to therapy even though their younger counterparts, as a group, tended to benefit even more.

Optimal dosage for aphasia therapy is an area of emerging focus in the field of aphasiology. A comprehensive review of this issue found insufficient evidence to recommend an optimal dosage that would result in maximum treatment outcome. ${ }^{61}$ Although it seems highly plausible that more aphasia therapy is better in almost any case of aphasia, treatment dose as a pre- dictor of outcome remains under-studied. For a comprehensive review of this issue, readers are referred to Harvey et al. ${ }^{61}$

\section{Individual therapy vs. group therapy}

The most common mode of SLT is one-on-one delivery where a single clinician, typically a speech-language pathologist, treats a single patient. Another mode of SLT delivery is group therapy, a tradition that was started as early as post World War II in Veterans Administration hospitals in the United States. Very limited data are available on the effectiveness of group SLT for aphasia, and third party reimbursers tend not to support it. ${ }^{62}$ Regardless of the mode, most persons with chronic aphasia do not receive an adequate amount of aphasia therapy to maximize recovery. Two sources of evidence are particularly important in this context: First, as discussed above, considerable evidence supports the utility of SLT for aphasia. ${ }^{16-18}$ Second, some studies show that more therapy is better, regardless of intensity (the amount per time unit). ${ }^{63,64}$ Unfortunately, recent data on the typical number of aphasia therapy sessions are lacking. One survey published 20 years ago indicated that patients with aphasia in the United States could expect to receive approximately 15 sessions of therapy. ${ }^{65}$ It is unlikely that the average number of therapy sessions has increased in the current healthcare climate. Thankfully, the number of aphasia centers that provide more intensive therapy and aphasia groups is on the rise (e.g., references $\left.^{66-68}\right)$. Yet, the number of individuals served by these centers represents a very small fraction of the two million who have chronic aphasia in North America. ${ }^{69}$ There are several reasons why individuals with chronic aphasia are underserved. Lack of third party reimbursement is undoubtedly a major barrier; ${ }_{i}^{69}$ however, other factors such as difficulty with transportation to and from therapy and limited availability of speech-language pathologists, especially in rural areas, may also play a role.

\section{In-person vs. telerehabilitation}

One way to improve access to aphasia therapy is by means of telerehabilitation (telerehab). Providing SLT via remote therapy could vastly increase the availability of speech-language pathologists and possibly decrease costs. A few studies have started to explore the effectiveness of telerehab for aphasia, both clinician-supervised therapy and unsupervised therapy (computerized therapy). One recently completed pilot trial indicated that supplementing 'standard of care' SLT for aphasia with telerehab did not improve the primary outcome (naming) compared to 'standard of care' SLT by itself. ${ }^{70}$ It is worth noting; however, that this trial was not aimed at establishing whether telerehab by itself is non-inferior to traditional in-person SLT. A separate trial compared the effects of telerehab and in-person 
SLT in individuals with chronic aphasia and found no difference between the two modes of therapy. ${ }^{71}$ Unfortunately, this study included only five participants in each condition and, therefore, was almost surely underpowered. A larger study ${ }^{72}(n=44)$ compared in-person SLT to a hybrid version of telerehab SLT: one session per week of clinician administered therapy via telerehab, supplemented with computerized therapy (Lingraphica) ${ }^{73}$ at least four times a week. This trial found hybrid telerehab to be non-inferior to in-person SLT for chronic aphasia. In a separate study, Telerehabilitation Group Aphasia Intervention and Networking (TeleGAIN), ${ }^{74} 19$ participants with chronic aphasia underwent group treatment for 12 weeks that was delivered via telerehab. Here, treatment focused on conversational engagement among participants with aphasia as well as functional treatment approaches. Although this study assessed a large number of endpoints, its most notable result was that participants communicative quality of life was improved after treatment compared to baseline. This is a very promising finding, which can be further tested in a larger trial that includes a control group and where the statistical analyses control for multiple comparisons when calculating changes across outcome measures.

Larger studies are needed to show that telerehab is non-inferior to in-person SLT, which will allow expanded availability of aphasia rehabilitation. The recent coronavirus disease 2019 (COVID-19) pandemic has forced many clinicians to transition to telerehab in place of face-to-face delivery, and third party payors have begun to cover the charges, which suggests a potential for this type of service delivery model to expand access to SLT for aphasia.

\section{Computerized therapy}

A fast-emerging approach to aphasia management is computerized SLT. One group study $(n=21)$ that employed a pre- and post-comparison of naming abilities showed a positive outcome of a 6-month SLT regimen. ${ }^{75}$ Much like many other trials, this study also showed a relationship between aphasia severity and long-term outcome with more severe patients maintaining less improvement at 4 months post-therapy. The authors concluded that independent home practice using computerized therapy could represent a viable alternative to clinician-based therapy, which often is not available to chronic patients. A large RCT $(n=278)$ with three arms further supports the potential utility of computerized aphasia therapy (Big CACTUS). ${ }^{76}$ Here, patients at least 4 months post-stroke were randomized to receive usual care; usual care supplemented with self-administered computerized SLT; or usual care with added attention therapy. The group that received the added computerized SLT experienced greater improvement in naming $(P<0.0001)$, but not in functional communication ( $P>0.05)$, compared to the other two groups. Although improvement in 'real life' functional communication is the desired outcome of aphasia therapy, such improvements are notoriously difficult to measure. Nevertheless, the results provide convincing evidence that computerized SLT may further enhance naming ability in aphasia. Subsequently, Latimer et al. ${ }^{77}$ analyzed the Big CACTUS data to understand whether adding self-administered computerized SLT to usual care was cost-effective. Unfortunately, because Big CACTUS did not yield a statistically significant improvement in quality of life associated with the addition of computerized SLT to usual care, the cost-effectiveness analysis was inconclusive. Nevertheless, this study emphasizes the need for other trials to follow suit and build in cost-effectiveness analyses so that stakeholders can understand not only whether a given treatment is effective in improving communication abilities but also whether it is cost-effective.

A more recently completed trial by Fleming et al. ${ }^{78}$ relied on a cross-over design to study the effects of computerized therapy to improve auditory comprehension in aphasia. Using an in-house developed app (Listen-In) that targets auditory comprehension at the single word, phrase, and sentence level and is run on a computer tablet, participants $(n=35)$ were randomized to first receive 12 weeks of self-administered Listen-In training and then 12 weeks of usual care, or vice versa. One of the several strengths of this trial was that participants received a relatively high dose of therapy with an average of 85 hours of training using Listen-In. A study-specific outcome test of auditory comprehension revealed greater comprehension improvement for trained items associated with Listen-In in comparison to usual care $(t(34)=4.09, P<0.001$, Cohen's $d=1.32)$. A similar comparison for untrained items revealed non-significant results. Changes in auditory comprehension measured on two sub-tests of the Comprehensive Aphasia Test ${ }^{79}$ were also not significant between Listen-In and usual care. Although not the purpose of the current review, it is notable that Fleming et al. ${ }^{78}$ also revealed structural brain changes (assessed using T1MRI) in both white (left hemisphere) and gray matter (right hemisphere) superior temporal gyrus that correlated with therapy outcome in a sub-set of the participants $(n=25)$. Although these effects need to be verified in a larger sample, this study provides convincing evidence that aphasia therapy changes the structure of the brain. Most importantly for clinical purposes, the study by Fleming et al. ${ }^{78}$ demonstrates that computerized therapy can improve auditory comprehension in aphasia. Other smaller studies also further support the utility of computerized SLT. For example, a study of seven chronic stroke patients with aphasia showed that computerized SLT yielded better language 
outcomes than a 'mind-game' intervention that did not focus on speech or language training. ${ }^{80}$

\section{Theory-based therapy}

There is a wide range in SLT approaches with regard to which mechanism they tackle or what aspects of language they are expected to improve. For example, most of the studies reviewed so far focus on speech production without necessarily emphasizing which aspect of processing is targeted by therapy. In contrast, Treatment of Underlying Forms (TUF) specifically emphasizes meta-linguistic training to improve grammatical processing, both for speech production and comprehension, in patients with Broca's aphasia. ${ }^{81}$ In short, TUF initially focuses on training lexical properties of verbs and mapping verb arguments. Then, training moves to complex sentence structures with emphasis on integration and reanalysis of semantic roles. One of the keys to TUF is that it trains more complex sentence structures, which has been shown to generalize to simpler structures. ${ }^{82}$ Although TUF is not a new therapy approach, a recent study with randomized assignment to either TUF $(n=14)$ or a control group that received no therapy $(n=5)$ provides evidence that TUF improves sentence processing among aphasic patients with agrammatism. ${ }^{83}$ Relying on the principles of TUF, training focused on complex sentence structures (long passive sentences with adjunct clauses), which resulted in improvements among the experimental group in both comprehension and production of trained sentences structures, as well as related sentences structures, at both immediate follow-up and at 3 months following therapy completion. No changes in performance were noted in the control group immediately after therapy completion, and no changes in the processing of unrelated sentence structures were noted in the experimental group. Along with several smaller studies, ${ }^{81,84}$ this study by Barbieri at al. ${ }^{83}$ suggests TUF as a preferred approach to treat patients with agrammatic aphasia.

In the past two decades, considerable research has focused on the effects of constraint-induced language therapy (CIAT) for aphasia. ${ }^{85}$ This behavioral therapy approach was based on evidence from motor rehabilitation indicating that constraining the spared limb and forcing patients to use the affected limb in therapy resulted in significant motor improvement. Applying this approach to SLT for aphasia, patients are discouraged from using modalities other than speech, such as gestures or writing, to communicate. This approach has shown promise in several trials, ${ }_{1}^{86,87}$ although it is not superior to more conventional SLT provided at similar intensity levels. ${ }^{88,89}$ It is possible that the success of CIAT so far is primarily related to relatively greater intensity of therapy in those studies rather than the actual constraining of non-verbal expression. An ongoing RCT
(Constraint-Induced or Multi-Modal Personalized Aphasia Rehabilitation [COMPARE]) by Rose et al..$^{90}$ may shed light on this issue by comparing the effectiveness of CIAT to multi-modality focused SLT as well as standard of care SLT. Unlike CIAT, the multi-modal SLT and the standard of care approaches do not incorporate the constraining aspects of CIAT, but therapy time is controlled across the three study arms. At the time this is written, enrollment in COMPARE is complete, but the results have not yet been published.

\section{Social approach to therapy}

We end this section by discussing what we consider a very positive development to managing aphasia: Life Participation Approaches to Aphasia (LPAA). Although LPAA is not a new concept, it has risen in popularity with annual conferences and workshops. Unlike impairment-based or functional therapy approaches to aphasia therapy that focus primarily on reestablishing communication abilities, LPAA emphasizes reintegration of persons with aphasia into the community by focusing on residual personal strengths instead of communication disabilities. LPAA could be regarded as an umbrella term for different tactics and approaches that maximize life participation. For example, training where individualized communication strategies are identified and practiced by the person with aphasia and communication partners can be effective. ${ }^{91-93}$ Along the same lines, testing for what individuals can, rather than cannot, communicate may help identify residual language abilities and potential communication strengths that otherwise might go unnoticed. ${ }^{94}$ Another important development in the LPAA realm is the increasing number of aphasia groups and aphasia centers. ${ }^{6795,96}$ Participation in aphasia groups and centers not only provides psycho-social benefits but may also improve communication and language. ${ }^{97}$ Despite the lack of large trials of the utility of LPAA, efforts focused on reintegration of persons with aphasia into society should be considered in very positive light. This brings us back to the issue of severity and the fact that persons with the most severe aphasia are unlikely to respond to SLT. We suggest that patients with the most severe aphasia, and, as a result, the least likely individuals to respond to SLT, may be best served by focusing more on LPAA, including counseling, and improving communication via approaches such as conversational coaching ${ }_{1}^{98}$ training alternative communication modalities, ${ }^{99}$ or even alternative or augmentative communication devices. ${ }^{100,101}$ This notion is developed in more detail below.

Several large RCTs of SLT in aphasia provide evidence that patients with more severe aphasia are less likely to respond to therapy. ${ }^{18,29,30}$ This effect is very salient and is demonstrated in Figure 1 where participants with the most severe aphasia 

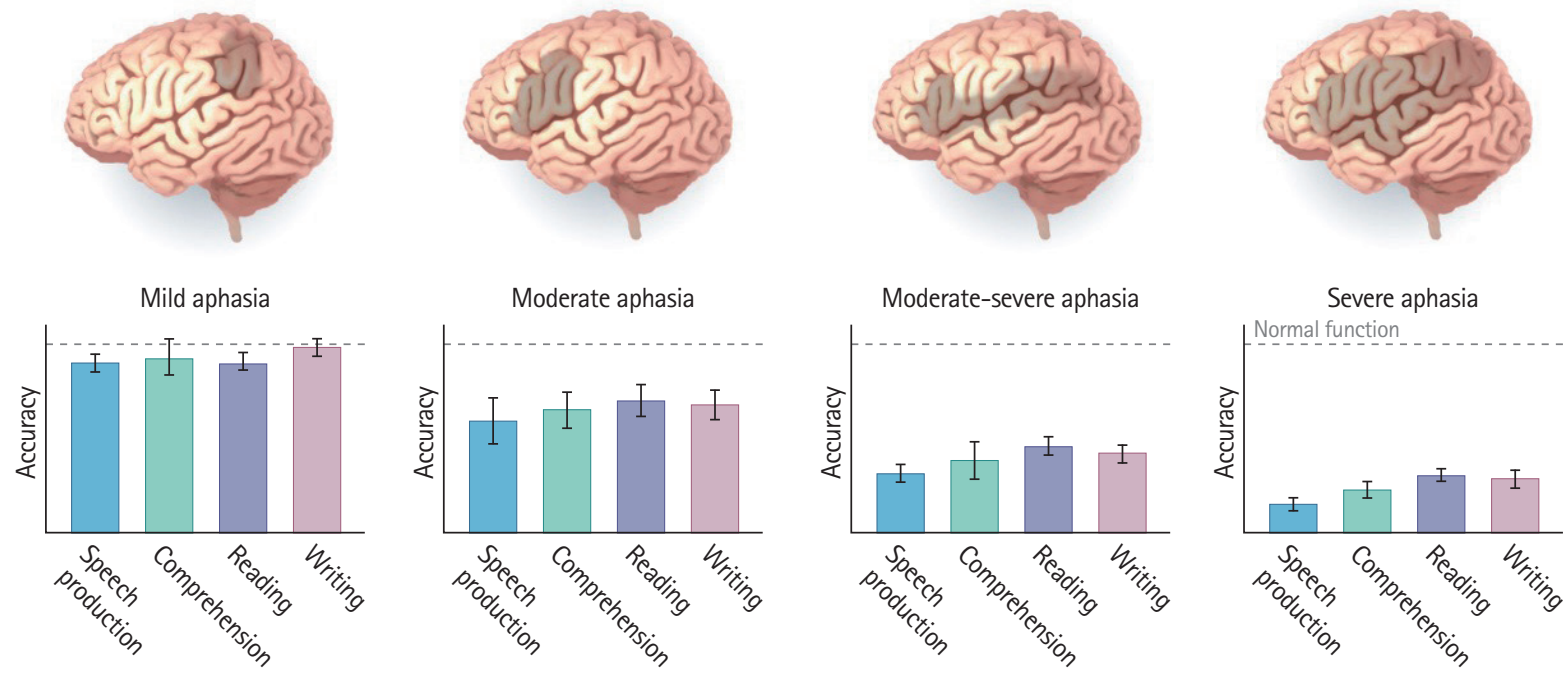

Impairment-based or functional SLT

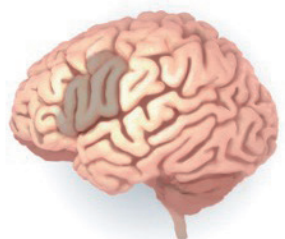
SLT

Figure 4. A simple schema suggesting how clinicians might determine how much of their effort in treatment should be devoted to speech and language therapy (SLT) versus Life Participation Approaches to Aphasia (LPAA) based on aphasia severity.

in the POLAR trial experienced very minimal improvement in speech production following 30 sessions of SLT. We suspect that the patients most likely to be referred for SLT are the most severe patients. Unfortunately, these individuals are the least likely to respond to SLT compared to their counterparts with moderate or mild aphasia. Based on this evidence, it would be tempting to discourage referrals of the most severe patients for SLT and, instead, focus rehabilitation on the less severe cases who are more likely to be responders. We think this would be a mistake as we believe all persons with aphasia would benefit from speech therapy services, but that these services need to be personalized based on the individuals' impairment profile, communicative needs, and recovery potential. In Figure 4, we suggest a simple rubric for how severity of aphasia might be used to determine how rehabilitation efforts could be best tailored to the needs of individuals patients. For the purpose of illustration, we have arranged aphasia severity levels from left-to-right starting with the mildest cases of aphasia, which usually involve relatively smaller cortical lesions, and progressing to the most severe cases on the right, which tend to have the most extensive cortical lesions. The graphs represent hypothetical language impairment scores for speech production, comprehension, reading, and writing. The bottom of the figure represents a proportional continuum between impairment or functional based therapy versus LPAA or counseling. The patients with the most severe aphasia, including those with global aphasia, would focus mostly on LPAA with relatively little effort on SLT. Those with less severe aphasia, primarily in the middle of the range between very severe and mild aphasia, would focus relatively less on LPAA but more on direct SLT using either impairment-based or functional SLT approaches. The patients with the least severe aphasia would focus almost all of their effort on direct SLT. Crucially, the type of rehabilitation and level of counseling would need to be further personalized within different levels of overall severity to maximize benefit. This last point cannot be overstated as overall severity levels can mask substantially different patterns of language impairment, personal needs, and goals among patients who score in the same severity range on aphasia tests.

\section{Pharmaceutical interventions}

\section{Acute interventions}

In acute ischemic stroke, the primary mechanism of recovery is restoration of blood flow to the penumbral tissue surrounding the core infarct. Numerous large RCTs have shown overall benefit in outcome with intravenous thrombolysis ${ }^{102}$ or endovascular therapy. ${ }^{103,104}$ While none of these trials have specifically evaluated the effects on language, case series studies have shown that such interventions do result in improvement of language functions. ${ }^{105}$ One small RCT also showed that temporary elevation of blood flow to improve perfusion early after left hemisphere stroke due to large vessel occlusion or stenosis was associated with language improvement. ${ }^{106}$ 
Pharmaceutical interventions to designed to augment behavioral language therapy for aphasia No study has provided evidence that pharmaceutical intervention results in significant improvement in language in chronic post-stroke aphasia in the absence of SLT. ${ }^{107}$ However, several trials have provided preliminary or inconsistent evidence that some medications may augment the effects of SLT, as reported below. A plausible mechanism is that language recovery depends on neuroplasticity-neural networks must be modified either by changing connectivity between undamaged nodes of the residual language network or by incorporating new nodes or other networks to assume the functions of the damaged ones. ${ }^{108,109}$ There is evidence from both humans and animals that behavioral interventions such as mass practice can bring about this type of reorganization through long-term potentiation and long-term depression. This type of neural plasticity is facilitated by the presence of neurotransmitters such as norepinephrine, acetylcholine, serotonin, and dopamine..$^{110,111}$ Therefore, medications that alter the availability of these neurotransmitters could enhance neuroplasticity. ${ }^{112}$ While most of the trials of medications to enhance recovery have focused on motor recovery, several RCTs have evaluated the effects on language recovery.

Early RCTs evaluated the effects of sympathomimetics, which elevate brain catecholamines. A few small nonrandomized trials (see Llano and Small ${ }^{113}$ ) and one larger RCT ${ }^{114}$ demonstrated small but statistically significant effects of dextroamphetamine in augmenting language therapy to improve language test scores. However, results were not adjusted for differences in language therapy duration and have not been subsequently replicated. Levadopa and bromocriptine have shown no consistent benefit over placebo in small RCTs. ${ }^{115-117}$ Likewise, small RCTs of cholinesterase inhibitors have shown only scant and/or short-lasting beneficial effects on language compared to placebo. ${ }^{11,119} \mathrm{Me}-$ mantine, a noncompetitive antagonist of the $\mathrm{N}$-methyl-D-aspartate (NMDA) receptor, with effects on serotonin and dopamine receptors and potential reduced excitotoxicity, ${ }^{120}$ has shown similar minor and short-lived positive effects in one open-label study, which has not been replicated in RCTs. ${ }^{13}$ Finally, selective serotonin reuptake inhibitors (SSRIs), which have been shown in RCTs to have a positive effect on post-stroke motor recovery measured with the Fugl Meyer scale ${ }^{121}$ and greater improvements on a cognitive battery, ${ }_{1}^{122}$ but no effect on the less sensitive modified Rankin Scale, ${ }^{123,124}$ have not been studied in RCTs for aphasia recovery. In summary, there is no consistent evidence that any medication has a substantial effect on aphasia recovery, despite numerous small RCTs to evaluate potential benefit conducted over the past five decades.

\section{Noninvasive brain stimulation}

Another approach to augmenting synaptic plasticity and reorganization of the networks that support language is through noninvasive brain stimulation. The two modalities that have been evaluated in RCT are transcranial direct current stimulation (tDCS) and repetitive transcranial magnetic stimulation (rTMS).

\section{tDCS}

The mechanisms underlying the effects of tDCS on function are not entirely clear, although studies have shown that application of weak ( 1 to $4 \mathrm{~mA}$ ) continuous electrical current to the scalp induces a subthreshold polarization of cortical neurons beneath the stimulated area. The polarization is too weak to generate an action potential, but "primes" the network that is stimulated by the concurrent behavioral task, altering their excitability. ${ }^{125}$ In this way, tDCS paired with a behavioral task can augment short- and long-term synaptic plasticity. Studies in animals and humans have provided evidence that tDCS effects depend on a brain derived neurotrophic factor (BDNF)-dependent mechanism. ${ }^{126,127}$ While the influence of the current on polarity of neurons is relatively widely distributed, functional MRI studies reveal that the entire network activated by the concurrent task is modulated when tDCS is applied to any part of the network, ${ }^{128}$ but the excitability is specific to the task-related network. Not surprisingly, then, tDCS applied in isolation (without concurrent language task) has no effect on language function in participants with aphasia. ${ }^{129}$

Double-blind RCTs of tDCS use a sham control that participants are unable to distinguish from real tDCS. The sham consists of 30 seconds of 1 to $2 \mathrm{~mA}$ stimulation which is faded to 0 , mimicking the sensation of continuous (e.g., 20 minutes) 1 to 2 $\mathrm{mA}$ stimulation, which is generally perceived for only the initial 20 to 30 seconds. ${ }^{129}$ RCTs in the last 5 years have evaluated 1 to $2 \mathrm{~mA}$ of anodal or cathodal tDCS for 15 to 20 minutes, based on rodent studies by Fritsch et al. ${ }^{126}$ demonstrating that 15 minutes of continuous tDCS significantly increases BDNF levels for longer than 1 hour. Anodal tDCS consistently increases the firing rate of targeted neurons, while cathodal tDCS has mixed effects. ${ }^{129}$ In humans, over 35 RCTs have been carried out to evaluate anodal or cathodal tDCS (or both) for post-stroke aphasia (see Biou et al. ${ }^{130}$ in 2019 for a systematic review). The location of electrode placement, language therapy methods, and number of sessions vary across RCTs, although most have used anodal tDCS over left hemisphere language areas, or anodal or cathodal tDCS over right hemisphere homologues (the latter with the goal to inhibit potentially adverse activity in the 
right hemisphere), or use left anodal and right cathodal simultaneously.

Most trials of tDCS have been carried out in chronic stroke, and most published studies have shown better language outcomes in the tDCS group or condition relative to the sham group or condition in each of these trials, even though both groups generally show some gains with SLT (with or without tDCS; see recent Cochrane review ${ }^{131}$ ). For example, one study demonstrated anodal tDCS over the left IFG in conjunction with language therapy resulted in significantly greater improvement compared to sham in picture description (gains of 19.5 vs. 10.6 correct words, $P=0.033$ ), noun naming (18.3 vs. $9.2, P=0.024$ ), and verb naming (18.4 vs. 7.3, $P=0.019) .{ }^{132}$ Several studies have reported that anodal tDCS (relative to sham) delivered to motor cortex paired with naming therapy results in greater gains in language outcomes. ${ }^{133,134}$ The largest $(n=74)$ double-blind RCT of tDCS to augment naming therapy reported significantly greater improvement in accuracy of naming untrained items with tDCS versus sham, each paired with a computer-delivered lexical-semantic therapy for 15 sessions. ${ }^{135}$ Improvement in naming was $70 \%$ greater in the left hemisphere anodal tDCS group relative to the sham group: a mean increase in accurately named objects from 13.9 (95\% confidence interval [Cl], 9.0 to 18.7) for tDCS versus 8.2 (95\% Cl, 3.8 to 12.6) for sham. Furthermore, there was an interaction between anodal tDCS and polymorphism of the BDNF gene. ${ }^{127}$ In the group that received tDCS, participants with the normal val/val polymorphism showed greater response to aphasia therapy than the Met allele carriers, whereas corresponding differences were not revealed among participants in the sham condition. ${ }^{127}$

Studies of cathodal tDCS to augment SLT have targeted right IFG or right cerebellum. A group of patients who received cathodal tDCS to right IFG with naming therapy showed faster response times in naming compared to the group who received sham plus naming therapy (1.29 to 2.57 seconds, $P=0.050){ }^{136}$ consistent with the hypothesis that inhibiting right IFG may facilitate language. ${ }^{137}$ A few studies have used cathodal (or anodal) tDCS to the right cerebellum with language therapy, based on the cerebellum's role in language and learning and both inhibitory and excitatory connections between the right cerebellum and left language cortex. ${ }^{138-140} A$ randomized order, double-blind, sham-controlled within-subject cross-over trial of right cerebellar tDCS showed greater improvements in spelling with $\mathrm{tDCS}$ versus sham (both with spelling therapy) on trained (from $0 \%$ to $52.5 \%$ correct vs. $0 \%$ to $97.5 \%$ correct, $P<0.05)$ and untrained words ( $0 \%$ to $27.5 \%$ correct vs. $0 \%$ to $82.5 \%$ correct, $P<0.01) .{ }^{138}$ Generalization to written picture naming was achieved only after right cerebellar anodal tDCS.

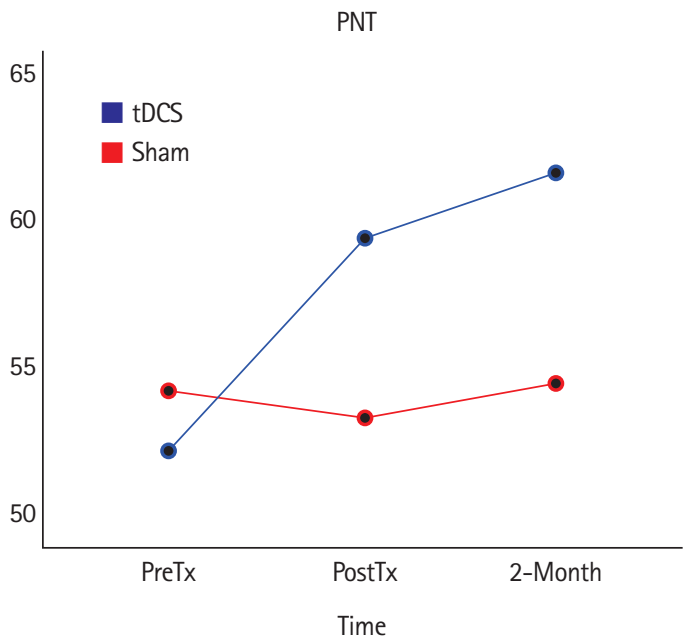

Figure 5. Mean accuracy in naming untrained words on the Philadelphia Naming Test (PNT) with right cerebellar transcranial direct current stimulation (tDCS) versus sham conditions, at pre-treatment (PreTx), post-treatment (PostTx), and 2 months PostTx. This figure illustrates data from reference 133 but combines data for anodal and cathodal right cerebellar tDCS, which did not have significantly different effects. Both tDCS conditions resulted in greater improvements than sham from PreTx to PostTx, and sustained 2 months later. To see results reported separately for anodal and cathodal tDCS, see reference 111.

More recent data from a randomized order, double-blind crossover trial of right cerebellar tDCS versus sham, combined with computer-delivered lexical-semantic therapy in 20 participants with chronic post-stroke aphasia by our collaborators showed significantly improved naming of treated items in both conditions, but improvement in untreated items only during the tDCS condition (Figure 5). ${ }^{139}$

The few sham-controlled studies of tDCS+SLT in subacute post-stroke aphasia have shown modest gains with right cathodal $\mathrm{tDCS}^{141}$ or no significant effect with just five therapy sessions. ${ }^{142}$ However, these studies have shown that 1 to $2 \mathrm{~mA}$ tDCS is well-tolerated and safe early after stroke. Additional RCTs in subacute stroke (with more than five therapy sessions) are ongoing.

\section{rTMS}

The rTMS is also believed to modulate neuronal activity and enhance neuroplasticity. Unlike tDCS, rTMS can cause action potentials (depending on the frequency), so it can result in changes in network connectivity strength in the absence of a behavioral task. High frequency rTMS is excitatory, while low frequency rTMS (usually $1 \mathrm{~Hz}$ ) is inhibitory. In trials to improve language, low frequency rTMS is generally applied to the contralesional right hemisphere to inhibit its activation, which is thought to enhance perilesional left hemisphere activation. ${ }^{137}$ Likewise, high frequency rTMS is generally applied to left hemi- 
sphere perilesional areas to enhance activation during language. Most rTMS studies include behavioral language intervention immediately after the stimulation. Most RCTs include a sham group or condition, although the conditions are usually distinguishable by participants who receive both conditions. Therefore, cross-over trials are not generally conducted (but see Rubi-Fessen et al. ${ }^{143}$ ), as the participants cannot be adequately blinded to the intervention condition. However, randomized, parallel, sham-controlled studies are possible.

In subacute stroke, studies of both low frequency and high frequency rTMS have reported benefits in language recovery. One recent RCT used high frequency rTMS to the left IFG and low frequency rTMS to the right IFG (or bilateral sham) for 10 sessions, each followed by 30 minutes of SLT. The rTMS group showed significantly greater improvements than the sham group in accuracy of word comprehension $(P=0.04)$, naming $(P=0.01)$, repetition ( $P=0.002)$, and in aphasia severity ( $1.8 \pm 1.2$ vs. $0.9 \pm 0.3$, $P=0.018) .{ }^{144}$ Significant gains in language with rTMS were observed immediately after therapy and 2 months later, and were specific to language. In a pilot, blinded assessor RCT of low frequency rTMS (vs. sham) applied to the right IFG for 30 minutes, followed by SLT for 45 minutes, for 10 sessions in 12 patients, the rTMS group showed higher language scores post-treatment (e.g., $50.3 \pm 28.3$ vs. $39.3 \pm 18.1$ ) on a 100 -point summary score, despite similar baseline scores. The authors reported a significant time by group interaction but no $P$-values or $\mathrm{Cls}$ were reported. ${ }^{145} \mathrm{~A}$ larger cross-over study $(\mathrm{n}=30)$ of low frequency rTMS (vs. sham) to the right IFG, each with subsequent 45 minutes of language therapy for 10 sessions, also showed greater improvement with rTMS compared to sham on object naming $(47.4 \pm 28.3$ vs. $35.3 \pm 30.1, P<0.05)$, object naming reaction time $(12.1 \pm 4.9$ vs. $13.9 \pm 5.1, P<0.01)$, action naming $(34.8 \pm 24.6$ vs. $25.9 \pm 20.4$, $P<0.01)$, and action naming reaction time ( $15.4 \pm 5.2$ vs. $15.4 \pm 5.7$, $P<0.01)$ immediately after therapy, and lasting up to 3 months, and showed gains in functional communication. ${ }^{146}$

Similar effects of both low and high frequency rTMS have been reported in studies of chronic post-stroke aphasia (see a Naeser et al. ${ }^{137}$ for review and Ren et al. ${ }^{147}$ for a meta-analysis). One recent study first used functional near infrared spectroscopy to determine which hemisphere showed greater activation during language, to determine the most appropriate frequency of rTMS to right IFG. ${ }^{148}$ Patients with greater left hemisphere language activation received low frequency (inhibitory) rTMS to contralesional right IFG and those with more right hemisphere language activation received high frequency (excitatory) rTMS to right IFG. All eight patients received language therapy following rTMS, for 10 sessions. Equally significant improvements in language functions were observed with low and high frequency rTMS to right IFG, but there was no sham control group or condition.

While most rTMS studies for aphasia include language therapy immediately after rTMS, one study investigated concurrent versus subsequent language therapy. ${ }^{149}$ They compared SLT immediately following $1 \mathrm{~Hz}$ rTMS to right IFG, to synchronous delivery of language therapy with rTMS, and to language therapy alone. Synchronous language therapy was associated with greater improvements in verbal description, object naming, and action naming than either rTMS with subsequent language therapy or sham plus language therapy. The loud clicking noise during rTMS limits the type of language therapy that can be

Language network reorganization after stroke treatment

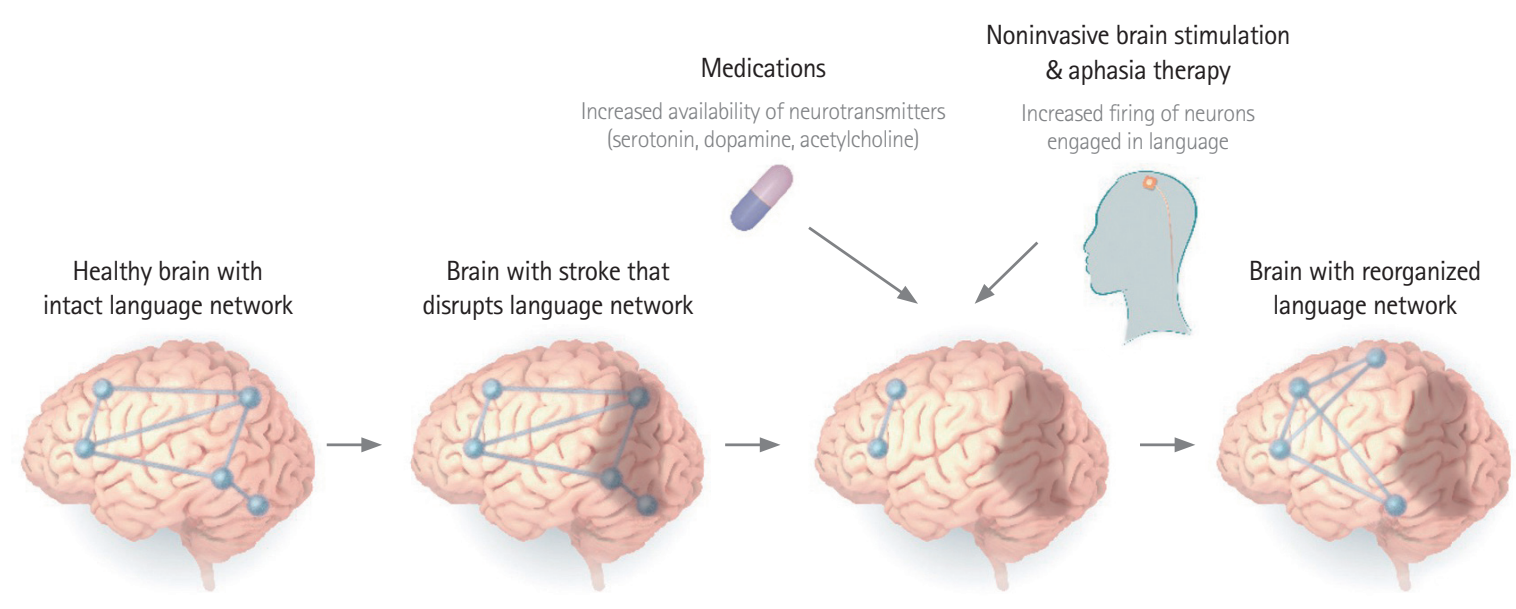

Figure 6. Combined behavioral, noninvasive brain stimulation, and pharmaceutical intervention promote reorganization of the language network to support improved language processing, as demonstrated through a variety of functional imaging studies of aphasia recovery with treatment. ${ }^{3-6,112}$ 
undertaken simultaneously with TMS.

\section{Conclusions}

The mainstay of post-stroke aphasia therapy has always been, and continues to be, behavioral SLT. There have been recent innovations to enhance its effectiveness, by increasing the time in therapy (e.g., through language therapy apps), by individualizing therapy to address aphasia severity and specific speech or language deficits, or increasing access to therapy through telerehab. Behavioral SLT has also been augmented with medications or noninvasive brain stimulation methods that facilitate neural plasticity. Functional imaging studies show changes in neural networks supporting language that correlate with improvements. Both animal studies and genetic studies of humans indicate that at least LDCS may work through a BDNF-dependent mechanism. Future studies will likely investigate the combination of pharmaceutical and noninvasive brain stimulation approaches to augment SLT. See Figure 6 for a schematic of how interventions lead to reorganization of the language network.

In spite of progress, a major priority for future research in aphasia rehabilitation is to establish 'minimum clinically significant differences' with regard to gains in both language and quality of life. Another important goal is to provide evidence for what therapy works best for patients with specific impairment and/or lesion profiles. A final goal is to identify the optimal dosage of aphasia therapy. Given that chronic aphasia is associated with especially poor quality of life, even worse than conditions such as cancer, ${ }^{150}$ we propose a more progressive research agenda aimed at larger trials with personally meaningful outcomes to establish best practices for aphasia rehabilitation.

\section{Disclosure}

The authors have no financial conflicts of interest.

\section{Acknowledgments}

The authors were supported by funding from NIH (NIDCD) through P50DC014664 and R01DC05375.

\section{References}

1. Nardo $D$, Holland R, Leff AP, Price $C J$, Crinion JT. Less is more: neural mechanisms underlying anomia treatment in chronic aphasic patients. Brain 2017;140:3039-3054.

2. Abel $\mathrm{S}$, Lambon Ralph MA. Cognitive neuroscience of aphasia recovery and therapy. Aphasiology 2018;32:739-741.
3. Crinion JT, Leff AP. Using functional imaging to understand therapeutic effects in poststroke aphasia. Curr Opin Neurol 2015;28:330-337.

4. Fridriksson J, Smith K. Neuroplasticity associated with treated aphasia recovery. In: Hickok G, Small SL. Neurobiology of Language. Amsterdam, NL: Elsevier, 2016;1007-1013.

5. Kiran S, Meier EL, Johnson JP. Neuroplasticity in aphasia: a proposed framework of language recovery. J Speech Lang Hear Res 2019;62:3973-3985.

6. Hartwigsen G, Saur D. Neuroimaging of stroke recovery from aphasia: insights into plasticity of the human language network. Neuroimage 2019;190:14-31.

7. Berker EA, Berker AH, Smith A. Translation of Broca's 1865 report: localization of speech in the third left frontal convolution. Arch Neurol 1986;43:1065-1072.

8. Fama ME, Turkeltaub PE. Treatment of poststroke aphasia: current practice and new directions. Semin Neurol 2014;34: 504-513.

9. Boyle M. Semantic feature analysis treatment for aphasic word retrieval impairments: what's in a name? Top Stroke Rehabil 2010;17:411-422.

10. Edmonds LA, Mammino K, Ojeda J. Effect of Verb Network Strengthening Treatment (VNeST) in persons with aphasia: extension and replication of previous findings. Am J Speech Lang Pathol 2014;23:S312-S329.

11. Kendall DL, Rosenbek JC, Heilman KM, Conway T, Klenberg K, Gonzalez Rothi $\sqcup$, et al. Phoneme-based rehabilitation of anomia in aphasia. Brain Lang 2008;105:1-17.

12. Martin N, Thompson CK, Worrall L. Aphasia Rehabilitation: The Impairment and Its Consequences. San Diego, CA: Plural Publishing, 2007.

13. Berthier ML, Green C, Lara JP, Higueras C, Barbancho MA, Dávila $\mathrm{G}$, et al. Memantine and constraint-induced aphasia therapy in chronic poststroke aphasia. Ann Neurol 2009;65: 577-585.

14. Kertesz A, McCabe P. Recovery patterns and prognosis in aphasia. Brain 1977;100 Pt 1:1-18.

15. Smania N, Gandolfi M, Aglioti SM, Girardi P, Fiaschi A, Girardi F. How long is the recovery of global aphasia?: twenty-five years of follow-up in a patient with left hemisphere stroke. Neurorehabil Neural Repair 2010;24:871-875.

16. Brady MC, Kelly H, Godwin J, Enderby P. Speech and language therapy for aphasia following stroke. Cochrane Database Syst Rev 2012;(5):CD000425.

17. Brady MC, Kelly H, Godwin J, Enderby P, Campbell P. Speech and language therapy for aphasia following stroke. Cochrane Database Syst Rev 2016;(6):CD000425.

18. Breitenstein C, Grewe T, Flöel A, Ziegler W, Springer L, Martus 
$\mathrm{P}$, et al. Intensive speech and language therapy in patients with chronic aphasia after stroke: a randomised, open-label, blinded-endpoint, controlled trial in a health-care setting. Lancet 2017;389:1528-1538.

19. Linebaugh CW, Baron CR, Corcoran KJ. Assessing treatment efficacy in acute aphasia: paradoxes, presumptions, problems and principles. Aphasiology 1998;12:519-536.

20. Robey RR. A meta-analysis of clinical outcomes in the treatment of aphasia. J Speech Lang Hear Res 1998;41:172-187.

21. Robey RR. The efficacy of treatment for aphasic persons: a meta-analysis. Brain Lang 1994;47:582-608.

22. Blomert L, Kean ML, Koster C, Schokker J. Amsterdam-Nijmegen everyday language test: construction, reliability and validity. Aphasiology 1994;8:381-407.

23. Doesborgh SJ, van de Sandt-Koenderman MW, Dippel DW, van Harskamp F, Koudstaal PJ, Visch-Brink EG. Effects of semantic treatment on verbal communication and linguistic processing in aphasia after stroke: a randomized controlled trial. Stroke 2004;35:141-146.

24. de Jong-Hagelstein $M$, van de Sandt-Koenderman WM, Prins ND, Dippel DW, Koudstaal PJ, Visch-Brink EG. Efficacy of early cognitive-linguistic treatment and communicative treatment in aphasia after stroke: a randomised controlled trial (RATS-2). J Neurol Neurosurg Psychiatry 2011;82:399-404.

25. Lincoln NB, McGuirk E, Mulley GP, Lendrem $W$, Jones AC, Mitchell JR. Effectiveness of speech therapy for aphasic stroke patients: a randomized controlled trial. Lancet 1984;1: 1197-1200.

26. de Courten-Myers GM, Kleinholz M, Wagner KR, Xi G, Myers RE. Efficacious experimental stroke treatment with highdose methylprednisolone. Stroke 1994;25:487-493.

27. Nouwens F, Visch-Brink EG, Van de Sandt-Koenderman MM, Dippel DW, Koudstaal PJ, de Lau LM. Optimal timing of speech and language therapy for aphasia after stroke: more evidence needed. Expert Rev Neurother 2015;15:885-893.

28. Warren RL, Gabriel C, Johnston A, Gaddie A. Efficacy during acute rehabilitation. Clinical Aphasiology Conference; 1987 May 31-Jun 4; Lake of the Ozarks, MO. Jackson, WY: BRK Publishers; 1-11.

29. Nouwens F, de Lau LM, Visch-Brink EG, van de Sandt-Koenderman WM, Lingsma HF, Goosen S, et al. Efficacy of early cognitive-linguistic treatment for aphasia due to stroke: a randomised controlled trial (Rotterdam Aphasia Therapy Study-3). Eur Stroke J 2017;2:126-136.

30. Godecke E, Armstrong E, Rai T, Ciccone N, Rose ML, Middleton $\mathrm{S}$, et al. A randomized control trial of intensive aphasia therapy after acute stroke: the Very Early Rehabilitation for SpEech (VERSE) study. Int J Stroke 2020 0ct 6 [Epub]. https:// doi.org/10.1177/1747493020961926.

31. Fridriksson J. Preservation and modulation of specific left hemisphere regions is vital for treated recovery from anomia in stroke. J Neurosci 2010;30:11558-11564.

32. Johnson L, Basilakos A, Yourganov G, Cai B, Bonilha L, Rorden $C$, et al. Progression of aphasia severity in the chronic stages of stroke. Am J Speech Lang Pathol 2019;28:639-649.

33. Holland A, Fromm D, Forbes M, MacWhinney B. Long-term recovery in stroke accompanied by aphasia: a reconsideration. Aphasiology 2017;31:152-165.

34. Hope TM, Leff AP, Prejawa S, Bruce R, Haigh Z, Lim L, et al. Right hemisphere structural adaptation and changing language skills years after left hemisphere stroke. Brain 2017; 140:1718-1728.

35. Basilakos A, Stark BC, Johnson L, Rorden C, Yourganov G, Bonilha $\mathrm{L}$, et al. Leukoaraiosis is associated with a decline in language abilities in chronic aphasia. Neurorehabil Neural Repair 2019;33:718-729.

36. Wilmskoetter J, Marebwa B, Basilakos A, Fridriksson J, Rorden $C$, Stark BC, et al. Long-range fibre damage in small vessel brain disease affects aphasia severity. Brain 2019;142: 3190-3201.

37. Levine DA, Galecki AT, Langa KM, Unverzagt FW, Kabeto MU, Giordani $\mathrm{B}$, et al. Trajectory of cognitive decline after incident stroke. JAMA 2015;314:41-51.

38. Darley FL. The efficacy of language rehabilitation in aphasia. J Speech Hear Disord 1972;37:3-21.

39. Borod JC, Carper JM, Naeser M. Long-term language recovery in left-handed aphasic patients. Aphasiology 1990;4:561572.

40. Laska AC, Hellblom A, Murray V, Kahan T, Von Arbin M. Aphasia in acute stroke and relation to outcome. J Intern Med 2001;249:413-422.

41. Pedersen PM, Vinter K, Olsen TS. Aphasia after stroke: type, severity and prognosis: the Copenhagen aphasia study. Cerebrovasc Dis 2004;17:35-43.

42. Engelter ST, Gostynski M, Papa S, Frei M, Born C, Ajdac-

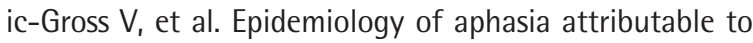
first ischemic stroke: incidence, severity, fluency, etiology, and thrombolysis. Stroke 2006;37:1379-1384.

43. Seniów J, Litwin M, Leśniak M. The relationship between non-linguistic cognitive deficits and language recovery in patients with aphasia. J Neurol Sci 2009;283:91-94.

44. Hope TM, Seghier ML, Leff AP, Price CJ. Predicting outcome and recovery after stroke with lesions extracted from MRI images. Neuroimage Clin 2013;2:424-433.

45. Selnes OA, Knopman DS, Niccum N, Rubens AB, Larson D. Computed tomographic scan correlates of auditory compre- 
hension deficits in aphasia: a prospective recovery study. Ann Neurol 1983;13:558-566.

46. Hillis AE, Beh YY, Sebastian R, Breining B, Tippett DC, Wright $A$, et al. Predicting recovery in acute poststroke aphasia. Ann Neurol 2018;83:612-622.

47. Marchina S, Zhu LL, Norton A, Zipse L, Wan CY, Schlaug G. Impairment of speech production predicted by lesion load of the left arcuate fasciculus. Stroke 2011;42:2251-2256.

48. Plowman $E_{1}$ Hentz B, Ellis C Jr. Post-stroke aphasia prognosis: a review of patient-related and stroke-related factors. J Eval Clin Pract 2012;18:689-694.

49. Cheng BB, Worrall LE, Copland DA, Wallace SJ. Prognostication in post-stroke aphasia: how do speech pathologists formulate and deliver information about recovery? Int J Lang Commun Disord 2020;55:520-536.

50. Spell LA, Richardson JD, Basilakos A, Stark BC, Teklehaimanot

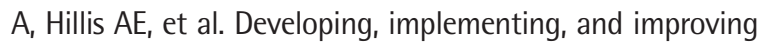
assessment and treatment fidelity in clinical aphasia research. Am J Speech Lang Pathol 2020;29:286-298.

51. Roach A, Schwartz MF, Martin N, Grewal RS, Brecher A. The Philadelphia naming test: scoring and rationale. Clin Aphasiol 1996;24:121-133.

52. Dignam J, Copland D, O'Brien K, Burfein P, Khan A, Rodriguez $A D$. Influence of cognitive ability on therapy outcomes for anomia in adults with chronic poststroke aphasia. J Speech Lang Hear Res 2017;60:406-421.

53. Gilmore N, Meier EL, Johnson JP, Kiran S. Nonlinguistic cognitive factors predict treatment-induced recovery in chronic poststroke aphasia. Arch Phys Med Rehabil 2019;100:12511258.

54. Kristinsson S, Basilakos A, Elm J, Spell LA, Bonilha L, Rorden C, et al. Individualized response to different types of aphasia rehabilitation. Brain Commun. 2021 In Press.

55. Fridriksson J, Yourganov G, Bonilha L, Basilakos A, Den Ouden $\mathrm{DB}$, Rorden $\mathrm{C}$. Revealing the dual streams of speech processing. Proc Natl Acad Sci U S A 2016;113:15108-15113.

56. Conroy P, Sotiropoulou Drosopoulou C, Humphreys GF, Halai $A D$, Lambon Ralph MA. Time for a quick word?: the striking benefits of training speed and accuracy of word retrieval in post-stroke aphasia. Brain 2018;141:1815-1827.

57. Bonilha L, Gleichgerrcht E, Nesland T, Rorden C, Fridriksson J. Success of anomia treatment in aphasia is associated with preserved architecture of global and left temporal lobe structural networks. Neurorehabil Neural Repair 2016;30:266-279.

58. Toth E, Gersner R, Wilf-Yarkoni A, Raizel H, Dar DE, Richter-Levin $G$, et al. Age-dependent effects of chronic stress on brain plasticity and depressive behavior. J Neurochem 2008; 107:522-532.
59. deToledo-Morrell L, Geinisman Y, Morrell F. Age-dependent alterations in hippocampal synaptic plasticity: relation to memory disorders. Neurobiol Aging 1988;9:581-590.

60. Vara H, Muñoz-Cuevas J, Colino A. Age-dependent alterations of long-term synaptic plasticity in thyroid-deficient rats. Hippocampus 2003;13:816-825.

61. Harvey S, Carragher M, Dickey MW, Pierce JE, Rose ML. Dose effects in behavioural treatment of post-stroke aphasia: a systematic review and meta-analysis. Disabil Rehabil 2020 Nov 9 [Epub]. https://doi.org/10.1080/09638288.2020.1843079.

62. Duchan JF, Byng S. Challenging Aphasia Therapies: Broadening the Discourse and Extending the Boundaries. Hove, UK: Psychology Press, 2004.

63. Carpenter J, Cherney LR. Increasing aphasia treatment intensity in an acute inpatient rehabilitation program: a feasibility study. Aphasiology 2016;30:542-565.

64. Dignam J, Copland D, McKinnon E, Burfein P, O'Brien K, Farrell $A$, et al. Intensive versus distributed aphasia therapy: a nonrandomized, parallel-group, dosage-controlled study. Stroke 2015;46:2206-2211.

65. Katz RC, Hallowell B, Code C, Armstrong E, Roberts P, Pound $C_{1}$ et al. A multinational comparison of aphasia management practices. Int J Lang Commun Disord 2000;35:303-314.

66. Hoover EL, Caplan DN, Waters GS, Carney A. Communication and quality of life outcomes from an interprofessional intensive, comprehensive, aphasia program (ICAP). Top Stroke Rehabil 2017;24:82-90.

67. Lanyon L, Worrall L, Rose M. Exploring participant perspectives of community aphasia group participation: from "I know where I belong now" to "some people didn't really fit in". Aphasiology 2018;32:139-163.

68. Ribeiro Lima R, Rose ML, Lima HD, Guarinello AC, Santos RS, Massi GA. Socio-demographic factors associated with quality of life after a multicomponent aphasia group therapy in people with sub-acute and chronic post-stroke aphasia. Aphasiology 2020 Feb 24 [Epub]. https://doi.org/10.1080/02687038. 2020.1727710 .

69. Simmons-Mackie N. Aphasia in North America. Moorestown, NJ: Aphasia Access, 2018.

70. Øra HP, Kirmess M, Brady MC, Partee I, Hognestad RB, Johannessen $B B$, et al. The effect of augmented speech-language therapy delivered by telerehabilitation on poststroke aphasia: a pilot randomized controlled trial. Clin Rehabil 2020;34:369381.

71. Woolf C, Caute A, Haigh Z, Galliers J, Wilson S, Kessie A, et al. A comparison of remote therapy, face to face therapy and an attention control intervention for people with aphasia: a quasi-randomised controlled feasibility study. Clin Rehabil 
2016;30:359-373.

72. Meltzer JA, Baird AJ, Steele RD, Harvey SJ. Computer-based treatment of poststroke language disorders: a non-inferiority study of telerehabilitation compared to in-person service delivery. Aphasiology 2018;32:290-311.

73. Steele RD. Lingraphic methods in interface design. In: Proceedings of the International Workshop on Human Interface Technology IWHIT 95; 1995 Oct 12-13; Aizu, JP: 53-58.

74. Pitt $R$, Theodoros $D$, Hill AJ, Russell T. The impact of the telerehabilitation group aphasia intervention and networking programme on communication, participation, and quality of life in people with aphasia. Int J Speech Lang Pathol 2019;21: 513-523.

75. Kurland J, Liu A, Stokes P. Effects of a tablet-based home practice program with telepractice on treatment outcomes in chronic aphasia. J Speech Lang Hear Res 2018;61:11401156.

76. Palmer R, Dimairo M, Cooper $C$, Enderby $P$, Brady M, Bowen $A$, et al. Self-managed, computerised speech and language therapy for patients with chronic aphasia post-stroke compared with usual care or attention control (Big CACTUS): a multicentre, single-blinded, randomised controlled trial. Lancet Neurol 2019;18:821-833.

77. Latimer NR, Bhadhuri A, Alshreef A, Palmer R, Cross E, Dimairo $M$, et al. Self-managed, computerised word finding therapy as an add-on to usual care for chronic aphasia poststroke: an economic evaluation. Clin Rehabil 2021;35:703717.

78. Fleming V, Brownsett S, Krason A, Maegli MA, Coley-Fisher $\mathrm{H}$, Ong $\mathrm{YH}$, et al. Efficacy of spoken word comprehension therapy in patients with chronic aphasia: a cross-over randomised controlled trial with structural imaging. J Neurol Neurosurg Psychiatry 2021;92:418-424.

79. Howard D, Swinburn K, Porter G. Putting the CAT out: what the Comprehensive Aphasia Test has to offer. Aphasiology 2010;24:56-74.

80. Stark BC, Warburton EA. Improved language in chronic aphasia after self-delivered iPad speech therapy. Neuropsychol Rehabil 2018;28:818-831.

81. Thompson CK, Shapiro LP. Treating agrammatic aphasia within a linguistic framework: treatment of underlying forms. Aphasiology 2005;19:1021-1036.

82. Murray L, Timberlake A, Eberle R. Treatment of underlying forms in a discourse context. Aphasiology 2007;21:139-163.

83. Barbieri E, Mack J, Chiappetta B, Europa E, Thompson CK. Recovery of offline and online sentence processing in aphasia: language and domain-general network neuroplasticity. Cortex 2019;120:394-418.
84. Thompson CK. Treatment of syntactic and morphologic deficits in agrammatic aphasia: Treatment of underlying forms. In: Chapey R. Language Intervention Strategies in Aphasia and Related Neurogenic Communication Disorders. 5th ed. Philadelphia, PA: Wolters Kluwer Health, 2012;735-755.

85. Zhang J, Yu J, Bao Y, Xie O, Xu Y, Zhang J, et al. Constraint-induced aphasia therapy in post-stroke aphasia rehabilitation: a systematic review and meta-analysis of randomized controlled trials. PLoS One 2017;12:e0183349.

86. Pulvermüller $F$, Neininger $B$, Elbert $T$, Mohr B, Rockstroh $B$, Koebbel $\mathrm{P}$, et al. Constraint-induced therapy of chronic aphasia after stroke. Stroke 2001;32:1621-1626.

87. Szaflarski JP, Ball AL, Vannest J, Dietz AR, Allendorfer JB, Martin AN, et al. Constraint-induced aphasia therapy for treatment of chronic post-stroke aphasia: a randomized, blinded, controlled pilot trial. Med Sci Monit 2015;21:28612869.

88. Sickert A, Anders LC, Münte TF, Sailer M. Constraint-induced aphasia therapy following sub-acute stroke: a single-blind, randomised clinical trial of a modified therapy schedule. $J$ Neurol Neurosurg Psychiatry 2014;85:51-55.

89. Ciccone N, West D, Cream A, Cartwright J, Rai T, Granger A, et al. Constraint-induced aphasia therapy (CIAT): a randomised controlled trial in very early stroke rehabilitation. Aphasiology 2016;30:566-584.

90. Rose ML, Copland D, Nickels L, Togher L, Meinzer M, Rai T, et al. Constraint-induced or multi-modal personalized aphasia rehabilitation (COMPARE): a randomized controlled trial for stroke-related chronic aphasia. Int J Stroke 2019;14:972-976.

91. Kagan A, Simmons-Mackie N, Victor JC. The impact of exposure with no training: implications for future partner training research. J Speech Lang Hear Res 2018;61:2347-2352.

92. Kagan A, Winckel J, Black S, Duchan JF, Simmons-Mackie N, Square $P$. A set of observational measures for rating support and participation in conversation between adults with aphasia and their conversation partners. Top Stroke Rehabil 2004; 11:67-83.

93. Simmons-Mackie N, Savage MC, Worrall L. Conversation therapy for aphasia: a qualitative review of the literature. Int J Lang Commun Disord 2014;49:511-526.

94. Holland A, Forbes M, Fromm D, MacWhinney B. Communicative strengths in severe aphasia: the famous people protocol and its value in planning treatment. Am J Speech Lang Pathol 2019;28:1010-1018.

95. Simmons-Mackie N, Holland AL. Aphasia centers in North America: a survey. Semin Speech Lang 2011;32:203-215.

96. Attard MC, Loupis Y, Togher L, Rose ML. Staff experiences of an interprofessional community aphasia group. Aphasiology 
2020;34:1-18.

97. Elman RJ. Aphasia centers and the life participation approach to aphasia. Top Lang Disord 2016;36:154-167.

98. Holland AL. Pragmatic aspects of intervention in aphasia. J Neurolinguist 1991;6:197-211.

99. Purdie $H$, Baldwin S. Music therapy: challenging low self-esteem in people with a stroke. J Br Music Ther 1994;8:19-24.

100. Nicholas M, Elliott S. C-Speak Aphasia: A Communication System for Adults with Aphasia. Solana Beach, CA: Mayer-Johnson Co., 1998.

101. Nicholas M, Sinotte M, Helm-Estabrooks N. Using a computer to communicate: effect of executive function impairments in people with severe aphasia. Aphasiology 2005;19: 1052-1065.

102. National Institute of Neurological Disorders and Stroke RTPA Stroke Study Group. Tissue plasminogen activator for acute ischemic stroke. N Engl J Med 1995;333:1581-1587.

103. Berkhemer OA, Fransen PS, Beumer D, van den Berg LA, Lingsma HF, Yoo AJ, et al. A randomized trial of intraarterial treatment for acute ischemic stroke. N Engl J Med 2015;372: 11-20.

104. Campbell BC, Mitchell PJ, Kleinig TJ, Dewey HM, Churilov L,

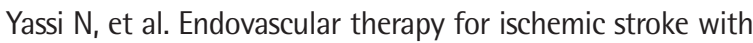
perfusion-imaging selection. N Engl J Med 2015;372:10091018.

105. Hillis AE. Pharmacological, surgical, and neurovascular interventions to augment acute aphasia recovery. Am J Phys Med Rehabil 2007;86:426-434.

106. Hillis $A E$, Ulatowski JA, Barker PB, Torbey M, Ziai W, Beauchamp NJ, et al. A pilot randomized trial of induced blood pressure elevation: effects on function and focal perfusion in acute and subacute stroke. Cerebrovasc Dis 2003;16:236246.

107. Berthier ML. Ten key reasons for continuing research on pharmacotherapy for post-stroke aphasia. Aphasiology 2020 May 31 [Epub]. https://doi.org/10.1080/02687038.2020.1769 987.

108. Crosson B, Rodriguez AD, Copland D, Fridriksson J, Krishnamurthy LC, Meinzer $M$, et al. Neuroplasticity and aphasia treatments: new approaches for an old problem. J Neurol Neurosurg Psychiatry 2019;90:1147-1155.

109. Stefaniak JD, Halai AD, Lambon Ralph MA. The neural and neurocomputational bases of recovery from post-stroke aphasia. Nat Rev Neurol 2020;16:43-55.

110. Brzosko Z, Mierau SB, Paulsen 0. Neuromodulation of spike-timing-dependent plasticity: past, present, and future. Neuron 2019;103:563-581.

111. Kirkwood A, Rozas C, Kirkwood J, Perez F, Bear MF. Modula- tion of long-term synaptic depression in visual cortex by acetylcholine and norepinephrine. J Neurosci 1999;19:15991609.

112. Kilgard MP, Merzenich MM. Cortical map reorganization enabled by nucleus basalis activity. Science 1998;279:17141718.

113. Llano DA, Small SL. Pharmacotherapy for aphasia. In: Hickok G, Small SL. Neurobiology of Language. Amsterdam, NL: Academic Press, 2015;1067-1083.

114. Walker-Batson D, Curtis S, Natarajan R, Ford J, Dronkers $N$, Salmeron $E_{\text {, et }}$ al. A double-blind, placebo-controlled study of the use of amphetamine in the treatment of aphasia. Stroke 2001;32:2093-2098.

115. Seniów J, Litwin $M$, Litwin $T$, Leśniak $M$, Członkowska A. New approach to the rehabilitation of post-stroke focal cognitive syndrome: effect of levodopa combined with speech and language therapy on functional recovery from aphasia. $J$ Neurol Sci 2009;283:214-218.

116. Ashtary $F$, Janghorbani $M$, Chitsaz $A$, Reisi $M$, Bahrami A. A randomized, double-blind trial of bromocriptine efficacy in nonfluent aphasia after stroke. Neurology 2006;66:914-916.

117. Breitenstein $C$, Korsukewitz $C$, Baumgärtner A, Flöel A, Zwitserlood $P$, Dobel $C$, et al. L-dopa does not add to the success of high-intensity language training in aphasia. Restor Neurol Neurosci 2015;33:115-120.

118. Berthier ML, Green $C_{1}$ Higueras $C$, Fernández I, Hinojosa J, Martín MC. A randomized, placebo-controlled study of donepezil in poststroke aphasia. Neurology 2006;67:16871689.

119. Haixia YE, Shilin LI. A clinical randomized case-control study of donepezil in the treatment of aphasia after stroke. Chin J Behav Med Brain Sci 2014;23:225-227.

120. Lipton SA. Paradigm shift in neuroprotection by NMDA receptor blockade: memantine and beyond. Nat Rev Drug Discov 2006;5:160-170.

121. Chollet F, Tardy J, Albucher JF, Thalamas C, Berard E, Lamy C, et al. Fluoxetine for motor recovery after acute ischaemic stroke (FLAME): a randomised placebo-controlled trial. Lancet Neurol 2011;10:123-130.

122. Jorge RE, Acion L, Moser D, Adams HP Jr, Robinson RG. Escitalopram and enhancement of cognitive recovery following stroke. Arch Gen Psychiatry 2010;67:187-196.

123. FOCUS Trial Collaboration. Effects of fluoxetine on functional outcomes after acute stroke (FOCUS): a pragmatic, double-blind, randomised, controlled trial. Lancet 2019;393:265274.

124. Kraglund KL, Mortensen JK, Damsbo AG, Modrau B, Simonsen $\mathrm{SA}$, Iversen $\mathrm{HK}$, et al. Neuroregeneration and vascular 
protection by citalopram in acute ischemic stroke (TALOS). Stroke 2018;49:2568-2576.

125. Bindman $\amalg$, Lippold OC, Redfearn JW. The action of brief polarizing currents on the cerebral cortex of the rat (1) during current flow and (2) in the production of long-lasting after-effects. J Physiol 1964;172:369-382.

126. Fritsch B, Reis J, Martinowich K, Schambra HM, Ji Y, Cohen LG, et al. Direct current stimulation promotes BDNF-dependent synaptic plasticity: potential implications for motor learning. Neuron 2010;66:198-204.

127. Fridriksson J, Elm J, Stark BC, Basilakos A, Rorden C, Sen S, et al. BDNF genotype and tDCS interaction in aphasia treatment. Brain Stimul 2018;11:1276-1281.

128. Holland R, Leff AP, Josephs O, Galea JM, Desikan M, Price CJ, et al. Speech facilitation by left inferior frontal cortex stimulation. Curr Biol 2011;21:1403-1407.

129. Crinion J. Transcranial direct current stimulation and aphasia therapy post stroke. In: Hillis AE. The Handbook of Adult Language Disorders. New York, NY; Psychology Press, 2015:458-475.

130. Biou $E_{1}$ Cassoudesalle $H$, Cogné $M$, Sibon I, De Gabory I, Dehail $\mathrm{P}$, et al. Transcranial direct current stimulation in poststroke aphasia rehabilitation: a systematic review. Ann Phys Rehabil Med 2019;62:104-121.

131. Elsner B, Kugler J, Pohl M, Mehrholz J. Transcranial direct current stimulation (tDCS) for improving aphasia in adults with aphasia after stroke. Cochrane Database Syst Rev 2019; 5:CD009760.

132. Campana $\mathrm{S}$, Caltagirone $\mathrm{C}$, Marangolo P. Combining voxel-based lesion-symptom mapping (VLSM) with A-tDCS language treatment: predicting outcome of recovery in nonfluent chronic aphasia. Brain Stimul 2015;8:769-776.

133. Darkow R, Martin A, Würtz A, Flöel A, Meinzer M. Transcranial direct current stimulation effects on neural processing in post-stroke aphasia. Hum Brain Mapp 2017;38:1518-1531.

134. Meinzer M, Darkow R, Lindenberg R, Flöel A. Electrical stimulation of the motor cortex enhances treatment outcome in post-stroke aphasia. Brain 2016;139(Pt 4):1152-1163.

135. Fridriksson J, Rorden C, Elm J, Sen S, George MS, Bonilha L. Transcranial direct current stimulation vs sham stimulation to treat aphasia after stroke: a randomized clinical trial. JAMA Neurol 2018;75:1470-1476.

136. Silva FR, Mac-Kay AP, Chao JC, Santos MD, Gagliadi RJ. Transcranial direct current stimulation: a study on naming performance in aphasic individuals. Codas 2018;30:e20170242.

137. Naeser MA, Martin PI, Treglia E, Ho M, Kaplan E, Bashir S, et al. Research with rTMS in the treatment of aphasia. Restor Neurol Neurosci 2010;28:511-529.
138. Sebastian R, Saxena $S$, Tsapkini $K$, Faria AV, Long C, Wright A, et al. Cerebellar tDCS: a novel approach to augment language treatment post-stroke. Front Hum Neurosci 2017;10: 695.

139. Sebastian R, Kim JH, Brenowitz R, Tippett DC, Desmond JE, Celnik PA, et al. Cerebellar neuromodulation improves naming in post-stroke aphasia. Brain Commun 2020;2:fcaa179.

140. Dvorak E, DeMarco A, Stoodley C, Turkeltaub P. A pilot study of right cerebellar tDCS as a therapeutic adjuvant in chronic aphasia after left-hemisphere stroke (P5.6-006). Neurology 2019;92(15 Suppl):P5.6-006.

141. You DS, Kim DY, Chun MH, Jung SE, Park SJ. Cathodal transcranial direct current stimulation of the right Wernicke's area improves comprehension in subacute stroke patients. Brain Lang 2011;119:1-5.

142. Spielmann K, van de Sandt-Koenderman WM, Heijenbrok-Kal MH, Ribbers GM. Transcranial direct current stimulation does not improve language outcome in subacute poststroke aphasia. Stroke 2018;49:1018-1020.

143. Rubi-Fessen I, Hartmann A, Huber W, Fimm B, Rommel T, Thiel $A$, et al. Add-on effects of repetitive transcranial magnetic stimulation on subacute aphasia therapy: enhanced improvement of functional communication and basic linguistic skills: a randomized controlled study. Arch Phys Med Rehabil 2015;96:1935-1944.

144. Khedr EM, Abo El-Fetoh N, Ali AM, El-Hammady DH, Khalifa $\mathrm{H}$, Atta $\mathrm{H}_{\text {, et al. Dual-hemisphere repetitive transcranial }}$ magnetic stimulation for rehabilitation of poststroke aphasia: a randomized, double-blind clinical trial. Neurorehabil Neural Repair 2014;28:740-750.

145. Haghighi M, Mazdeh M, Ranjbar N, Seifrabie MA. Further evidence of the positive influence of repetitive transcranial magnetic stimulation on speech and language in patients with aphasia after stroke: results from a double-blind intervention with sham condition. Neuropsychobiology 2017;75: 185-192.

146. Tsai PY, Wang CP, Ko JS, Chung YM, Chang YW, Wang JX. The persistent and broadly modulating effect of inhibitory rTMS in nonfluent aphasic patients: a sham-controlled, double-blind study. Neurorehabil Neural Repair 2014;28:779787.

147. Ren $\mathrm{CL}$, Zhang GF, Xia N, Jin $\mathrm{CH}$, Zhang XH, Hao JF, et al. Effect of low-frequency rTMS on aphasia in stroke patients: a meta-analysis of randomized controlled trials. PLoS One 2014;9:e102557.

148. Hara T, Abo M, Kakita K, Mori Y, Yoshida M, Sasaki N. The effect of selective transcranial magnetic stimulation with functional near-infrared spectroscopy and intensive speech 
therapy on individuals with post-stroke aphasia. Eur Neurol 2017;77:186-194.

149. Wang CP, Hsieh CY, Tsai PY, Wang CT, Lin FG, Chan RC. Efficacy of synchronous verbal training during repetitive transcranial magnetic stimulation in patients with chronic apha- sia. Stroke 2014;45:3656-3662.

150. Lam JM, Wodchis WP. The relationship of 60 disease diagnoses and 15 conditions to preference-based health-related quality of life in Ontario hospital-based long-term care residents. Med Care 2010;48:380-387. 